\title{
Chimeric Hepatitis B core antigen virus-like particles displaying the envelope domain III of dengue virus type 2
}

Upasana Arora, Poornima Tyagi, Sathyamangalam Swaminathan* and Navin Khanna*

\begin{abstract}
Background: Dengue is a global public health problem for which no drug or vaccine is available. Currently, there is increasing interest in developing non-replicating dengue vaccines based on a discrete antigenic domain of the major structural protein of dengue viruses (DENVs), known as envelope domain III (EDIII). The use of bionanoparticles consisting of recombinant viral structural polypeptides, better known as virus-like particles (VLPs), has emerged as a potential platform technology for vaccine development. This work explores the feasibility of developing nanoparticles based on E. coli-expressed recombinant Hepatitis B virus core antigen ( $\mathrm{HBCAg}$ ) designed to display EDIII moiety of DENV on the surface.
\end{abstract}

Findings: We designed a synthetic gene construct encoding HBCAg containing an EDIII insert in its c/e1 loop. The fusion antigen HBCAg-EDIII-2 was expressed in E. coli, purified to near homogeneity using $\mathrm{Ni}^{+2}$ affinity chromatography and demonstrated to assemble into discrete 35-40 nm VLPs by electron microscopy. Competitive ELISA analyses showed that the EDIII-2 moieties of the VLPS are accessible to anti-EDIII-2-specific monoclonal and polyclonal antibodies, suggesting that they are surface-displayed. The VLPs were highly immunogenic eliciting high titer anti-EDIII-2 antibodies that were able to recognize, bind and neutralize infectious DENV based on ELISA, immunofluorescence and virus-neutralization assays.

Conclusion: This work demonstrates that HBcAg-derived nanoparticles can serve as a useful platform for the display of DENV EDIII. The EDIII-displaying nanoparticles may have potential applications in diagnostics/vaccines for dengue.

\section{Findings}

Dengue is a mosquito-borne viral disease prevalent in more than a hundred tropical and sub-tropical countries placing about 2.5 billion of the global population at risk, causing 50-100 million infections and $\sim 12,500$ deaths each year [1]. There are four serotypes of dengue viruses (DENV-1, -2, -3 and -4), belonging to the family Flaviviridae [2], each of which can cause dengue disease [1]. The tremendous challenges being faced in the development of live viral dengue vaccines [3] has spurred a keen interest in new generation non-replicating subunit vaccines [3,4]. In this context, a discrete domain of the viral envelope protein has been identified as a potential

\footnotetext{
* Correspondence: swami@icgeb.res.in; navin@icgeb.res.in

Recombinant Gene Products Group, International Centre for Genetic Engineering \& Biotechnology, Aruna Asaf Ali Marg, New Delhi 110067, India
}

subunit vaccine candidate [3-5]. This domain known as envelope domain III (EDIII), is exposed and accessible on the virion surface [6], contains multiple type- and subtype-specific neutralizing epitopes [7] and is implicated in host receptor binding [8].

One way to augment the vaccine potential of EDIII would be to display it in multiple copies on the surface of a nanoparticle carrier. Naturally occurring bionanomaterials, by virtue of their biocompatibility and biodegradability, are emerging as preferred platforms for biomedical applications [9]. Viruses are naturally occurring nanoparticles whose particulate nature with repetitive coat protein architecture and pathogen associated molecular patterns make them a potentially valuable platform for vaccine development. A subclass of viral nanoparticles can be generated in heterologous expression systems exploiting the propensity of several 
recombinant viral coat proteins to self-assemble. These genome-free viral nanoparticles which eliminate the infectious biohazard inherent in the naturally occurring viral nanoparticles are more popularly known as viruslike particles (VLPs) $[9,10]$. The 183 amino acid (aa) residue Hepatitis B virus core antigen ( $\mathrm{HBcAg}$ ) which can assemble into VLPs is a well-documented carrier of foreign antigens from several different pathogens [11] and HBcAg-based VLP vaccine candidates for malaria and influenza are being evaluated in clinical trials $[12,13]$. Of relevance to our study is the reported capacity of $\mathrm{HBcAg}$ to accommodate foreign inserts (in the size range of 100-260 aa residues) in its surface-exposed c/e1 loop, with retention of its VLP-forming ability. This loop which contains the major B-cell epitopes ' $\mathrm{c}$ ' and ' $\mathrm{e} 1$ ' is also known as the major immunodominant region of the HBcAg VLPs [11]. Optimal immunogenicity of the foreign insert is ensured by the concomitant removal of these major $\mathrm{HBcAg}$ immunodominant epitopes. Interestingly, many of these chimeric VLPs have been produced using E. coli expression hosts [14-20]. In this study, we report the design, creation and characterization of chimeric nanoparticles containing DENV-2 EDIII (herein indicated as EDIII-2) inserted into the c/e1 loop of HBcAg. Further we show that these chimeric VLPs display EDIII-2 on the surface and elicit antibodies specific to DENV-2.

The chimeric HBcAg-EDIII-2 antigen was designed by replacing aa residues $76-80$ in the c/e1 loop of a C- terminally truncated $\mathrm{HBcAg}$ molecule (lacking aa residues 166 to 183 ) with the 104 aa residue EDIII-2. We introduced a spacer (GSGDEGG) between the C-terminus of the EDIII-2 insert and aa 81 of $\mathrm{HBcAg}$ to minimize any disruption of particle assembly through potential interactions between $\beta$-sheet forming residues of EDIII- 2 and aa 80-90 of HBcAg [21]. To aid in purification the chimeric antigen design included an N-terminal 6x His tag linked through a pentaglycyl spacer to the N-terminal end of $\mathrm{HBcAg}$. A synthetic gene, $H B c A g-E D I I I-2$, encoding this chimeric fusion antigen (GenBank accession \# JQ729976), codon-optimized for E. coli expression, was inserted into an IPTG-inducible prokaryotic expression vector (Figure 1A and Additional file 1: Figure S1). E. coli transformed with this plasmid, expressed the fusion antigen upon induction, (Figure 1B). The identity of this induced protein band was confirmed using antibodies specific to each of the two fusion partners as well as to the affinity tag by immunoblotting analyses (Figure 1C).

A localization analysis of the induced cell lysate revealed the fusion antigen to be associated exclusively with the insoluble fraction (Figure 2A). This is consistent with the behavior of a multitude of heterologous proteins over-expressed in E. coli. Attempts to obtain the fusion antigen in the soluble phase by performing the IPTG induction at lower temperatures failed. While induction at $16{ }^{\circ} \mathrm{C}$ did not result in discernible expression of the recombinant antigen, at $25{ }^{\circ} \mathrm{C}$ it was barely discernible. Expression was evident at higher induction
A

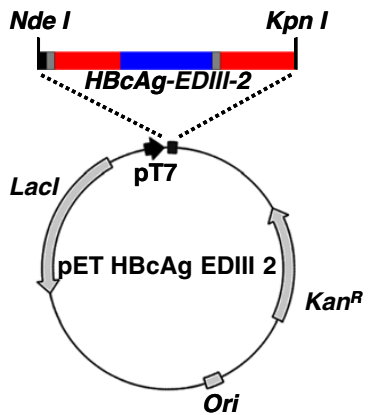

B

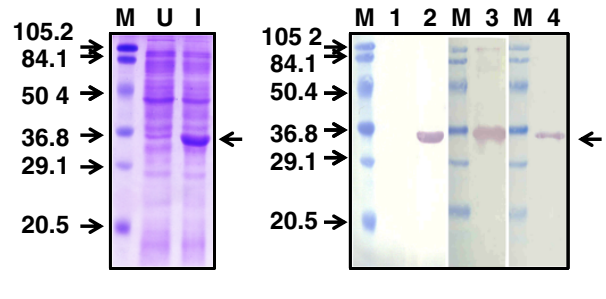

Figure 1 Design and expression of HBCAg-EDIII-2 antigen in E. coli. (A) A map of the HBCAg-EDIII-2 expression vector. The synthetic HBCAgEDIII-2 gene is inserted under the control of the phage T7 promoter (pT7) in PET29a. The organization of different segments of this fusion gene is indicated in colour as follows. The HBcAg- and EDIII-2-encoding regions are shown in red and blue, respectively. The 6x His tag-encoding sequences at the 5 'end is shown in black. The two linker-encoding sequences, the first following the $6 \times$ His tag and the second after the EDIII-2 encoding sequences, are shown in grey. Other abbreviations are as follows. Lac I: Lac repressor gene; Kan ${ }^{R}$ : Kanamycin marker; Ori: Replication origin sequences. (B) SDS-PAGE analysis of recombinant HBCAg-EDIII-2 expression. This panel displays the Coomassie-stained polypeptide profiles of lysates prepared from un-induced (U) and induced (I) E. coli cells harboring the plasmid shown in A. Pre-stained protein molecular weight markers were run in lane ' $\mathrm{M}$ '. Their sizes (in $\mathrm{kDa}$ ) are shown at the left of the panel. The arrow on the right indicates the position of the recombinant HBCAg-EDIII-2 protein. (C) Immunoblot analyses of recombinant protein expression. Aliquots of un-induced and induced cell lysates (described in panel 'B') were electrophoresced, electroblotted onto nitrocellulose membranes and probed with anti-EDIII mAb 24A12 (lane 2), penta His mAb (lane 3), or anti-HBcAg mAb ab8638 (lane 4). An aliquot of the un-induced cell lysate was probed with mAb 24A12 (lane 1). Prestained protein size markers were run in lanes marked ' $\mathrm{M}$ '. Their sizes (in $\mathrm{kDa}$ ) are indicated to the left of the first blot. The arrow to the right indicates the position of the recombinant HBcAg-EDIII-2 protein. 
temperatures (Additional file 1: Figure S2), but the expressed protein tended to be associated with the insoluble fraction. Consequently, we purified the protein from induced cells under denaturing conditions (Figure 2B). An imidazole step-gradient elution resulted in the emergence of two protein peaks, 1 and 2. Interestingly, an SDS-PAGE analysis of the two peaks revealed them to contain a single polypeptide co-migrating with the $\sim 30 \mathrm{kDa}$ low molecular weight marker. This mobility is consistent with the predicted size $(\sim 31 \mathrm{kDa})$ of the HBcAg-EDIII-2 antigen. As both peaks in Figure 2B appeared to be similar (Figure 2C), we pooled the two and dialyzed the material $(0.3 \mathrm{mg} / \mathrm{ml})$ against $25 \mathrm{mM}$ sodium bicarbonate buffer, $\mathrm{pH}$ 9.2. We could recover $\sim 95 \%$ of the protein after dialysis. Analysis of an aliquot of this by electron microscopy revealed the presence of discrete VLPs of $\sim 35-40 \mathrm{~nm}$ (Figure 3A). Interestingly, these were quite similar to purified, E. coli-expressed VLPs (Figure 3B). It is noteworthy that the HBcAgEDIII-2 protein had been purified under highly denaturing conditions. Yet, the dialyzed protein could be observed to be organized into VLPs. This underscores the fact that inherent propensity of $\mathrm{HBcAg}$ to selfassemble into VLPs is not compromised by the insertion
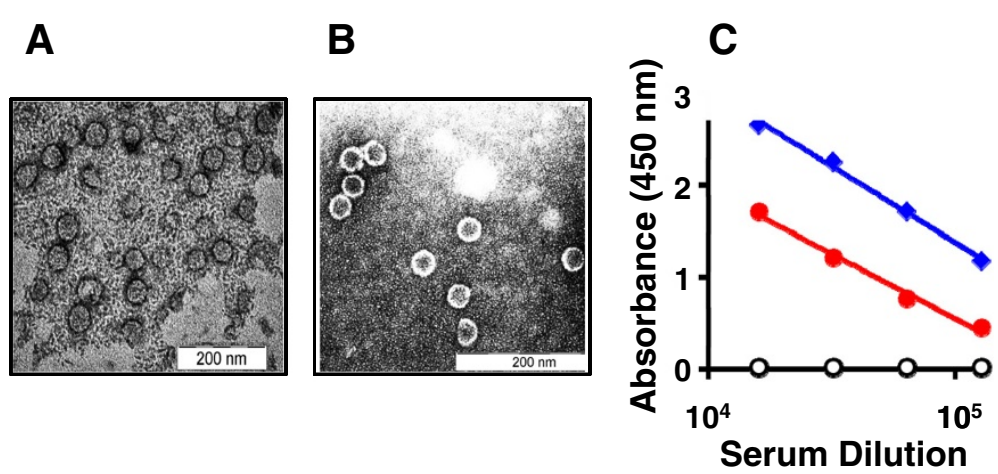

Figure 3 Characterization of the purified HBcAg-EDIII-2 antigen. (A) The panel depicts VLPs formed by purified HBCAg-EDIII-2 protein (expressed in P.pastoris). Peaks 1 and 2 (shown in Figure 2B) were pooled, dialyzed, stained with $1 \%$ uranyl acetate and visualized under the electron microscope. (B) Electron microscopic visualization of the purified HBcAg carrier (expressed in E. coli). (C) Evaluation of the immunogenicity of the HBCAg-EDIII-2 antigen by ELISA. Antisera from mice immunized with the fusion antigen (solid red circles) and its precursors (HBcAg: empty black circles; EDIII-2: solid blue diamonds) were analyzed in an ELISA using recombinant EDIII-2 antigen (produced using P. pastoris) as the coating antigen. 


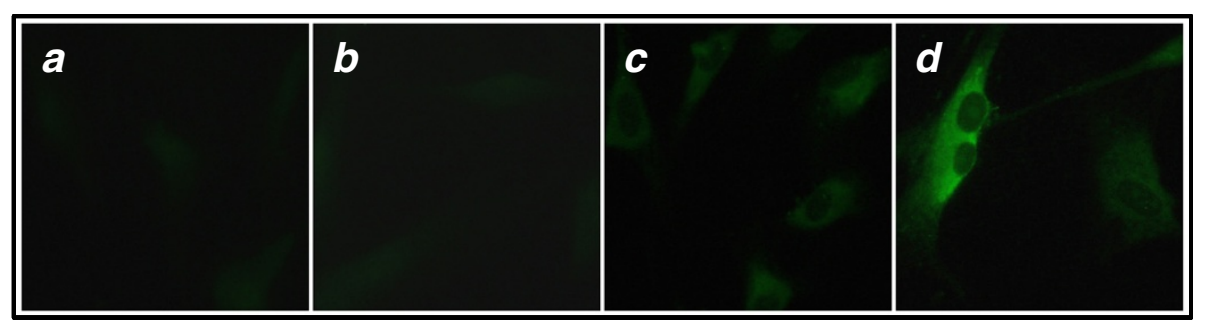

Figure 4 Evaluation of antibodies elicited by HBcAg-EDIII-2 fusion antigen by indirect immunofluorescence assay. Sera from mice that were mock-immunized (panel a), and immunized with HBCAg (panel b), EDIII-2 (panel c) or HBCAg-EDIII-2 (panel $d$ ) antigens were used as the primary antibody to pick up DENV-2 in infected BHK-21 cells, in conjunction with an anti-mouse lgG-FITC conjugate.

of EDIII-2 into its c/e1 loop. Starting from a liter of induced $E$. coli culture we obtained $\sim 7 \mathrm{mg}$ of $\mathrm{HBcAg-}$ EDIII-2 VLPs (Additional file 1: Table S1).

Next, we examined if these VLPs display the EDIII-2 to the immune system and induce specific antibodies. To this end, we immunized Balb/C mice with these VLPs and tested the immune sera for anti-EDIII-2 antibodies in an indirect ELISA using purified EDIII-2 [22] as the coating antigen. In parallel, we also tested sera from mice immunized with purified EDIII-2 and $\mathrm{HBcAg}$ antigens. The data in Figure $3 \mathrm{C}$ reveal that the fusion antigen does indeed elicit high titers of anti-EDIII-2 antibodies, lending support to the notion that the chimeric VLPs do indeed facilitate display of EDIII-2 efficiently to the immune system. However, the antiEDIII-2 antibody titers elicited by the fusion antigen appeared to be slightly but consistently lower than those elicited by the EDIII- 2 antigen. This we believe could be a reflection of the $\sim 3$ fold higher concentration of EDIII2 antigen compared to the HBcAg-EDIII-2 fusion antigen, per immunization dose, as the latter is $\sim 3$ times larger in size compared to the former. This leads to the suggestion that the anti-EDIII-2 antibody titers elicited by the two antigens may indeed be quite comparable. The next question we addressed was, would these antibodies also recognize and bind to infectious DENV-2. For this, we infected BHK cells with DENV-2 and probed them with anti-HBcAg-EDIII-2 antiserum, in conjunction with a secondary antibody FITC conjugate. The indirect immunofluorescence data in Figure 4 show that the antibodies elicited by the fusion antigen were as good as those elicited by EDIII-2, in picking up DENV-2 in infected BHK cells. We next performed a virus neutralization assay to determine the titers of DENV-2 virus-neutralizing antibody titers in these antisera using plaque reduction neutralization test (PRNT) as described previously [23]. The $\mathrm{PRNT}_{50}$ titers elicited by EDIII- 2 and the HBcAgEDIII-2 fusion antigens were, respectively, 10 and 35.

The above data suggest that the fusion antigen VLPs display the EDIII-2 moiety as expected. Then, it must be accessible to antigen combining sites on anti-EDIII mAbs and polyclonal antibodies. We tested this using a competitive ELISA approach, the results of which are shown in Figure 5. In this experiment we used three different antibodies, an EDIII-specific mAb 24A12 [22], a murine polyclonal antiserum obtained by immunization with a chimeric antigen containing the EDIIIs of all 4 DENVs linked in a tandem array [24], and the murine polyclonal antiserum raised using the HBcAg-EDIII-2 antigen. Remarkably, in all three cases, we observed that

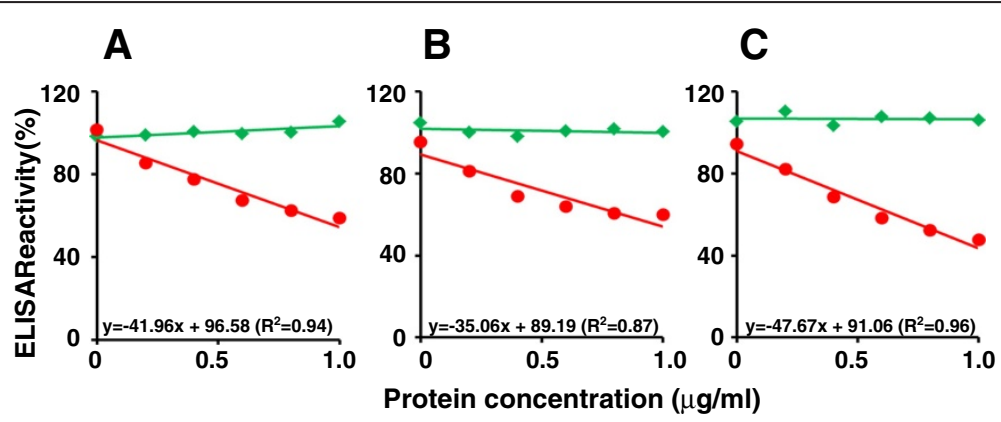

Figure 5 Competitive ELISA. Anti-EDIII mAb24A12 (panel A), anti-EDIII-T antiserum (panel B) and anti-HBCAg-EDIII-2 antiserum (panel C) were separately pre-incubated with varying concentrations of purified HBCAg (green curves) and HBCAg-EDIII-2 (red curves) VLPs, and analyzed for residual anti-EDIII-2 antibodies using purified P. pastoris-expressed EDIII-2 antigen as the coating antigen. ELISA reactivity in the absence of any added protein in the pre-incubation step was taken to represent 100\%. The regression equations for the HBCAg-EDIII-2 competition assays (red curves) are shown just above the horizontal axis in each panel. 
pre-incubation of antibodies with the HBcAg-EDIII-2 antigen, but not with the HBcAg antigen (lacking EDIII-2), resulted in a dose-dependent depletion of antigencombining sites available to bind EDIII-2 antigen coating the microtiter wells. These observations strongly support the notion that the chimeric VLPs do display the EDIII-2 moiety on the surface in a manner that is compatible with recognizing and binding to specific anti-EDIII-2 antibodies.

In conclusion, this work has demonstrated the feasibility of using an E. coli expression system to produce chimeric nanoparticles using a fusion antigen comprising of the HBcAg polypeptide with EDIII-2 inserted into its surface-exposed c/e1 loop. It has shown further that the EDIII-2 moiety is displayed on the surface of the chimeric nanoparticle and is able to induce the production of specific antibodies capable of binding and neutralizing the infectivity of DENV-2. This work provides the basis for us to envisage next generation $\mathrm{HBcAg-}$ derived mosaic nanoparticles that display the EDIII domains of not one, but all four DENV serotypes. Such nanoparticles could have potentially interesting and possibly useful diagnostic and vaccine potential.

\section{Additional file}

Additional file 1: The file is organized into 3 sections. Section S1 describes essential Methods. Section S2 provides supplementary figures pertaining to the fusion antigen design and sequence (Figure S1) and the effect of induction temperature on HBCAg-EDIII-2 expression (Figure S2). Section S3 provides a summary of HBCAg-EDIII-2 purification (Table S1).

\section{Abbreviations \\ aa: Amino acid; BHK: Baby hamster kidney; DENV: Dengue virus; DENV- 2: Dengue virus type 2; EDIII: Envelope domain III, EDIII-2, Envelope domain III of dengue virus type 2; ELISA: Enzyme-linked immunosorbent assay; FITC: Fluorescene isothiocyanate; HBCAg: Hepatitis B virus core antigen; mAb: Monoclonal antibody; SDS-PAGE: Sodium dodecyl sulfate- polyacrylamide gel electrophoresis; VLP: Virus-like particle.}

\section{Competing interests}

The authors declare that they have no competing interests.

\section{Acknowledgements}

SS and NK acknowledge the funding support received from the Department of Biotechnology, Government of India. UA was the recipient of a research fellowship from the Department of Biotechnology, Government of India.

\section{Authors' contributions}

SS and NK conceived and designed the study. UA and PT performed experiments. UA, SS and NK prepared the manuscript. All authors read and approved the manuscript.

Received: 1 May 2012 Accepted: 13 July 2012

Published: 13 July 2012

\section{References}

1. World Health Organization Factsheet No117: Dengue and dengue haemorrhagic fever, 2012. www.who.int/mediacentre/factsheets/fs117/en/ [Accessed April 27, 2012].
2. Gubler DJ, Kuno G, Markoff L: Flaviviruses. In Fields Virology. 5th edition. Edited by Knipe DM, Howley PM. Philadelphia: Wolters Kluwer and Lippincott Williams \& Wilkins; 2007:1153-1252.

3. Swaminathan S, Batra G, Khanna N: Dengue vaccines: state of the art. Expert Opin Ther Patents 2010, 20:819-835.

4. Schmitz J, Roehrig J, Barrett A, Hombach J: Next generation dengue vaccines: a review of candidates in preclinical development. Vaccine 2011, 29:7276-7284

5. Guzman MG, Hermida L, Bernardo L, Ramirez R, Guillén G: Domain III of the envelope protein as a dengue vaccine target. Expert Rev Vaccines 2010, 9:137-147.

6. Kuhn RJ, Zhang W, Rossman MG, Pletnev SV, Corver J, Lenches E, Jones CT, Mukhopadhyay S, Chipman PR, Strauss EG, Baker TS, Strauss JH: Structure of dengue virus: implications for flavivirus organization, maturation, and fusion. Cell 2002, 108:717-725.

7. Sukupolvi-Petty S, Austin SK, Purtha WE, Oliphant T, Nybakken GE, Schlesinger JJ, Roehrig JT, Gromowski GD, Barrett AD, Fremont DH, Diamond MS: Type- and subcomplex-specific neutralizing antibodies against domain III of dengue virus type 2 envelope protein recognize adjacent epitopes. J Virol 2007, 81:12816-12826.

8. Crill WD, Roehrig RT: Monoclonal antibodies that bind to domain III of dengue virus $\mathrm{E}$ glycoprotein are the most efficient blockers of virus adsorption to Vero cells. J Virol 2001, 75:7769-7773.

9. Yildiz I, Shukla S, Steinmetz NF: Applications of viral nanoparticles in medicine. Curr Opin Biotechnol 2011, 22:901-908.

10. Bachmann MF, Jennings GT: Vaccine delivery: a matter of size, geometry, kinetics and molecular patterns. Nat Rev Immunol 2010, 10:787-796

11. Pumpens $P$, Grens $E: H B V$ core particles as a carrier for $B$ cell/T cell epitopes. Intervirology 2001, 44:98-114.

12. Gregson AL, Oliveira G, Othoro C, Calvo-Calle JM, Thorton GB, Nardin ER, Edelman R: Phase I trial of an alhydrogel adjuvanted hepatitis B core virus-like particle containing epitopes of Plasmodium falciparum circumsporozoite protein. PLOS One 2008, 3:e1556.

13. Fiers W, de Filette M, Bakkouri KE, Schepens B, Roose K, Schotsaert M, Birkett A, Saelens X: M2e-based universal influenza A vaccine. Vaccine 2009, 27:6280-6283.

14. Kratz PA, Böttcher B, Nassal M: Native display of complete foreign protein domains on the surface of hepatitis B virus capsids. Proc Natl Acad Sci USA 1999, 96:1915-1920.

15. Wang XJ, Gu K, Xiong QY, Shen L, Cao RY, Li MH, Li TM, Wu J, Liu JJ: A novel virus-like particle-based on hepatitis $B$ core antigen and substratebinding domain of bacterial molecular chaperone DnaK. Vaccine 2009, 27:7377-7384

16. Skamel C, Ploss M, Böttcher B, Stehle T, Wallich R, Simon MM, Nassal M: Hepatitis B virus capsid-like particles can display the complete, dimeric outer surface protein $C$ and stimulate production of protective antibody response against Borrelia burgdorferi infection. J Biol Chem 2006, 281:17474-17481.

17. Nassal M, Skamel C, Kratz PA, Wallich R, Stehle T, Simon MM: A fusion product of the complete Borrelia burgdorferi outer surface protein $A$ (OspA) and the hepatitis B virus capsid protein is highly immunogenic and induces protective immunity similar to that seen with an effective lipidated OspA vaccine formula. Eur J Immunol 2005, 35:655-665.

18. Boulter NR, Glass EJ, Knight PA, Bell-Sakyi L, Brown CGD, Hall R: Theileria annulata sporozoite antigen fused to hepatitis $B$ core antigen used in a vaccination trial. Vaccine 1995, 13:1152-1160.

19. Koletzki D, Biel SS, Meisel H, Nugel E, Gelderblom HR, Krüger DH, Ulrich R: $\mathrm{HBV}$ core particles allow the insertion and surface exposure of the entire potentially protective region of Puumala hantavirus nucleocapsid protein. Biol Chem 1999, 380:325-333.

20. Geldmacher A, Skrastina D, Petrovskis I, Borisova G, Berriman JA, Roseman AM, Crowther RA, Fischer J, Musema S, Gelderblom HR, Lundkvist A, Renhofa $\mathrm{R}$, Ose V, Krüger DH, Pumpens P, Ulrich R: An amino-terminal segment of hantavirus nucleocapsid protein presented on hepatitis $B$ virus core particles induces a strong and highly cross-reactive antibody response in mice. Virology 2004, 323:108-119.

21. Karpenko LI, Ivanisenko VA, Pika IA, Chikaev NA, Eroshkin AM, Veremeiko TA, Ilyichev AA: Insertion of foreign peptides in $\mathrm{HBCAg}$ : how to make the chimeric particle assemble. Amino Acids 2000, 18:329-337. 
22. Batra G, Raut R, Dahiya S, Kamran N, Swaminathan S, Khanna N: Pichia pastoris-expressed dengue virus type 2 envelope domain III elicits virusneutralizing antibodies. J Virol Meth 2010, 167:10-16.

23. Korrapati AB, Swaminathan G, Singh A, Khanna N, Swaminathan S: Adenovirus Delivered Short Hairpin RNA Targeting a Conserved Site in the $5^{\prime}$ Non-Translated Region Inhibits All Four Serotypes of Dengue Viruses. PLoS Negl Trop Dis 2012, 6: e1735.

24. Etemad B, Batra G, Raut R, Dahiya S, Khanam S, Swaminathan S, Khanna N: An envelope domain III-based chimeric antigen produced in Pichia pastoris elicits neutralizing antibodies against all four dengue virus serotypes. Amer J Trop Med Hyg 2008, 79:353-363.

doi:10.1186/1477-3155-10-30

Cite this article as: Arora et al:: Chimeric Hepatitis B core antigen viruslike particles displaying the envelope domain III of dengue virus type 2 . Journal of Nanobiotechnology 2012 10:30.

\section{Submit your next manuscript to BioMed Central and take full advantage of:}

- Convenient online submission

- Thorough peer review

- No space constraints or color figure charges

- Immediate publication on acceptance

- Inclusion in PubMed, CAS, Scopus and Google Scholar

- Research which is freely available for redistribution 